\title{
On the secondary $\mathrm{X}$-ray emission induced by electron irradiation in thin samples
}

\author{
Eric Van Cappellen $\left({ }^{1}\right)$, Rudy Deblieck $\left({ }^{2}\right)$ and Dirk Van Dyck $\left({ }^{1}\right)$ \\ ( ${ }^{1}$ ) University of Antwerp, RUCA, Groenenborgerlaan 171, B-2020 Antwerp, Belgium \\ ( ${ }^{2}$ ) DSM Research, Postbus 18, NL-6160 Geleen, The Netherlands \\ (Received April 24, 1990; accepted July 03, 1990)
}

\begin{abstract}
Résumé. - Une expression générale pour l'émission secondaire de rayons-X induite par électrons dans des composés binaires est dérivée. Des simulations sur ordinateur utilisant cette expression ont été faites pour des échantillons cunéiformes, cette géometrie étant la plus fréquente en microscopie en transmission conventionelle et à balayage. Ceci a permit d'étudier la fluorescence caractéristique en fonction de l'épaisseur de l'échantillon tout en changeant la géometrie de la cible (l'angle du coin) ainsi que le potentiel d'accélération du microscope. Les influences de l'orientation de l'échantillon dans le faisceau d'électrons et de la direction de détection des rayons-X ont aussi été analysées. Ces calculs ont permis de constater que la géometrie de l'échantillon influence fortement la fluorescence caractéristique alors que les autres paramètres mentionnés plus haut n'ont qu'une importance secondaire.
\end{abstract}

\begin{abstract}
In the present paper earlier results in the field of secondary X-ray emission in binary compounds are reviewed and extended. A general expression for the $\mathrm{X}$-ray fluorescence emission in electron excited (scanning) transmission electron microscopy ((S) TEM) specimens is derived. Based on this expression computer simulations are carried out for wedge shaped targets, so as to investigate characteristic fluorescence emission as a function of specimen thickness, wedge angle and primary accelerating voltage and this for various electron impact directions and $\mathrm{X}$-ray detection directions. The general conclusion is that a small change in specimen geometry can have a serious influence on the secondary emission intensity.
\end{abstract}

\section{Introduction.}

Primary X-radiation generated by an electron beam in a specimen will partially be absorbed by the specimen. This process in turn generates characteristic X-radiation, the so-called secondary or fluorescence emission. Two components can be distinguished : continuum fluorescence caused by continuum primary radiation and characteristic fluorescence generated by a primary characteristic line. In X-ray microanalysis it is not possible to distinguish primary from secondary radiation. As a consequence the characteristic lines present in a spectrum will be larger than predicted by theoretical models taking only primary radiation into account. Whenever important fluorescence 
contributions are generated, the results of a quantitative analysis should be corrected for the fluorescence effects.

Tixier [1] and Philibert et al. [2] developed a formula for characteristic fluorescence correction for plane parallel foils. Their equation states that the fluorescence intensity is proportional with $T^{2}$ where $T$ is the mass thickness of the foil. Tixier [1] also demonstrated that continuum fluorescence is some orders of magnitude smaller than characteristic fluorescence since Bremstrahlung only represents typically 1 to 2 weight \% of the whole spectrum. (Statham [3] ). It is generally accepted that for a (S) TEM specimen continuum fluorescence is always negligible also because Bremsstrahlung is mainly produced in the forward direction when referred to the electron beam.

A second characteristic fluorescence correction formula for plane parallel foils was developed by Nockolds et al. [4]. The mass thickness dependance is totally different from that of the previous model and is proportional with $T .\left|0.923-\ell \mathrm{n}\left(\mu_{\mathrm{B}} \cdot T\right)\right|$ where $\mu_{\mathrm{B}}$ is the mass absorption coefficient of the exciting line in the specimen. According to Nockolds et al. [4] the Tixier formula strongly underestimates characteristic fluorescence especially in the "thin foil" range.

Both correction procedures were developed for plane parallel foils. However, an important influence of the real geometry of the specimen on the fluorescence intensity cannot be excluded, especially since secondary radiation can be generated far away (sometimes more than $1 \mathrm{~mm}$ ) from the primary excitation volume due to the large mean free paths of $\mathrm{X}$-rays. Apart from a few exceptions no (S) TEM specimen can be considered as plane parallel on the submillimeter scale. Therefore it is interesting to investigate whether characteristic fluorescence is sensitive to the geometry of the specimen. For this purpose, an infinite wedge with a wedge angle $\alpha$ is chosen to perform the computer simulations. This choice was inspired by the fact that most selfsupporting thinned (S) TEM specimens are more or less wedge shaped in the neighbourhood of the hole and also because it is the easiest shape to handle mathematically. If the geometry has an important influence this will certainly show up from calculations based on a wedge model.

To avoid a useless overload of the mathematical formalism the model will be developed for a binary $\mathrm{A}_{X} \mathrm{~B}_{1-X}$ compound in which a spectral line of one element (e.g. $\mathrm{B}$ ) is able to excite the other. The results of such a model can always be extended to more elements.

It should be emphasized in this introduction that the goal of this paper is merely to examine the fluorescence behaviour in an infinite wedge when the tip is irradiated by a fine electron probe and not provide a fluorescence correction procedure as such. Hence the choice of the composition ( $\mathrm{Cu} 50$ at \% - Cr) of the object used for simulation is deliberately not very fluorescing.

For a discussion about the relative magnitude of the absorption and the fluorescence we refer to the previous paper [9] .

\section{Secondary X-ray emission.}

2.1 Model (VAN CAPPELLEN [5] ). - A binary compound $A_{X} B_{1-X}$ is considered in which element "A" can be excited by primary characteristic radiation of element " $B$ ". In other words, at least one characteristic line of element " $B$ "is able to excite a line of element " $A$ ". A cross-section of the situation to be described is represented in figure 1 . The specimen is thinned so as to be suitable for transmission or scanning transmission electron microscopy. The mass thickness of the specimen along the optical axis of the microscope and the primary excitation volume are respectively denoted $T$ and $V$, whereas $C_{\mathrm{A}}$ and $C_{\mathrm{B}}$ represent the mass concentrations of the elements $\mathrm{A}$ and $\mathrm{B}$, respectively. The primary emission of the element $\mathrm{B}$ in the element of volume $\mathrm{d} V$ situated at a point $P(\mathbf{r})$ can be written as follows :

$$
\mathrm{d} I_{\mathrm{B}}^{0}=D C_{\mathrm{B}} \varphi(\mathbf{r}) \mathrm{d} V
$$




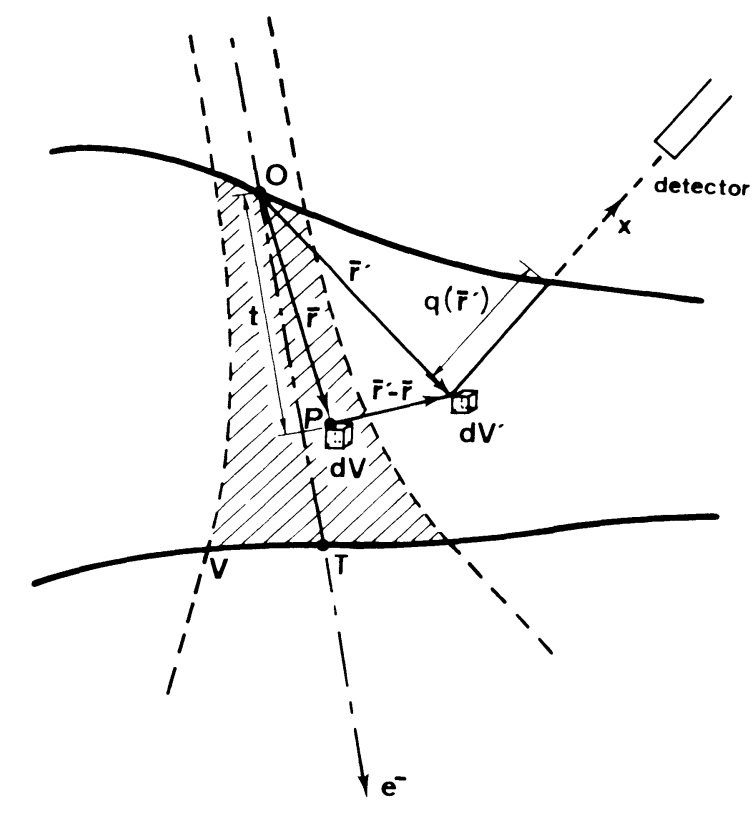

Fig. 1. - Cross-section of the model used to calculate the secondary emission of element $\mathrm{A}$ induced by primary emission of element B. Primary emission generated in $\mathrm{d} V$ will induce secondary emission in $\mathrm{d} V^{\prime}$. Integration over the specimen volume $\left(\mathrm{d} V^{\prime}\right)$ and subsequently over the excitation volume $V(\mathrm{~d} V)$ yields the total amount of secondary emission of element $\mathrm{A}$.

$\varphi(\mathbf{r})$ is the spacial ionization distribution function of element B and the constant $D$ is essentially the fluorescence yield $\omega_{\mathrm{B}}^{i}$ of the excited $i$-shell of element B multiplied with the weight factor $W_{\mathrm{B}}^{\delta}$ of the spectral $\delta$-line under consideration. Note that all distances are expressed in mass-length units. The characteristic radiation intensity $\mathrm{d} I_{\mathrm{B}}^{0}$ is emitted isotropically throughout the specimen. The fraction of this characteristic radiation reaching $\mathrm{d} V^{\prime}$ at position $\mathbf{r}^{\prime}$ is then given by (see Fig. 1):

$$
\mathrm{d} I_{\mathrm{B}}=\frac{\mathrm{d} \Omega}{4 \pi} \exp \left(-\mu_{\mathrm{B}}\left|\mathbf{r}^{\prime}-\mathbf{r}\right|\right) \mathrm{d} I_{\mathrm{B}}^{0}
$$

where $\mathrm{d} \Omega$ is the solid angle spanned by volume $\mathrm{d} V^{\prime}$ as seen from position $\mathbf{r}^{\prime}$ in $\mathrm{d} V$ and $\mu_{\mathrm{B}}$ the mass absorption coefficient of the considered line of element $B$. A fraction of this intensity will be absorbed by the A-atoms present in the element of volume $\mathrm{d} V^{\prime}$. If the mass absorption coefficient of the characteristic B-line in a pure element $\mathrm{A}$ target is denoted $\mu_{\mathrm{B}}^{\mathrm{A}}$, the absorbed primary intensity $\mathrm{d} I_{\mathrm{B}}^{\mathrm{A}}$ reads :

$$
\mathrm{d} I_{\mathrm{B}}^{\mathrm{A}}=C_{\mathrm{A}} \mu_{\mathrm{B}}^{\mathrm{A}} \mathrm{d}\left(\mathbf{r}^{\prime}-\mathbf{r}\right) \mathrm{d} I_{\mathrm{B}}
$$

This absorbed primary radiation then gives rise to fluorescence radiation given by :

$$
\mathrm{d} I_{\mathrm{A}}^{\mathrm{f}}=\frac{r_{\mathrm{A}}^{j}-1}{r_{\mathrm{A}}^{j}} \omega_{\mathrm{A}}^{j} W_{\mathrm{A}}^{\gamma} \mathrm{d} I_{\mathrm{B}}^{\mathrm{A}}
$$

The first factor $\left(r_{\mathrm{A}}^{j}-1\right) / r_{\mathrm{A}}^{j}$ is the ionization yield with $r_{\mathrm{A}}^{j}$ the absorption edge jump ratio, (Philibert et al. [2] ; Nockolds et al. [4] ). $\omega_{\mathrm{A}}^{j}$ is the fluorescence yield of the excited $j$-shell of element A and $W_{\mathrm{A}}^{\gamma}$ is the weight factor of that line. 
Only a small fraction of this intensity will contribute to the detected signal, namely the intensity reaching the surface of the specimen in the direction of the detector. This intensity $\mathrm{d} I_{\mathrm{A}}^{\mathrm{F}}$ reads :

$$
\mathrm{d} I_{\mathrm{A}}^{\mathrm{F}}=\frac{\mathrm{d} \Omega^{\prime}}{4 \pi} \exp \left(-\mu_{\mathrm{A}} q\left(\mathbf{r}^{\prime}\right)\right) \mathrm{d} I_{\mathrm{A}}^{\mathrm{f}}
$$

where $\mu_{\mathrm{A}}$ is by analogy the mass absorption coefficient of the $\gamma$-line of element $\mathrm{A}$ in the examined sample. $\mathrm{d} \Omega$ ' and $q\left(\mathbf{r}^{\prime}\right)$ respectively stand for the solid angle spanned by the detector and the distance between the element of volume $d V^{\prime}$ and the surface of the specimen in the direction of the detector (Fig. 1). This distance $q\left(\mathbf{r}^{\prime}\right)$ is a function of the position $\mathbf{r}^{\prime}$ of $\mathrm{d} V^{\prime}$ but also of the overall geometry of the specimen and the detector direction. For a general specimen geometry the behaviour of $q\left(\mathbf{r}^{\prime}\right)$ will be extremely complicated and this limits the final choice of a geometry to a tractable one.

The total secondary $\gamma$-line radiation of element $\mathrm{A}, I_{\mathrm{A}}^{\mathrm{F}}$ generated by primary $\delta$-line radiation of element $\mathrm{B}$ and reaching the detector is found by integrating the element of volume $\mathrm{d} V^{\prime}$ over the specimen volume and subsequently the element of volume $\mathrm{d} V$ over the excitation volume $V$ :

$$
I_{\mathrm{A}}^{\mathrm{F}}=\int_{V} \int_{\text {spec. }} \mathrm{d} I_{\mathrm{A}}^{\mathrm{F}}
$$

or :

$$
I_{\mathrm{A}}^{\mathrm{F}}=C \int_{V} \int_{\text {spec. }} \varphi(\mathbf{r}) \frac{\exp \left(-\mu_{\mathrm{B}}\left|\mathbf{r}^{\prime}-\mathbf{r}\right|\right)}{\left|\mathbf{r}^{\prime}-\mathbf{r}\right|^{2}} \exp \left(-\mu_{\mathrm{A}} q\left(\mathbf{r}^{\prime}\right)\right) \mathrm{d} V^{\prime} \mathrm{d} V
$$

where the constant $C$ equals :

$$
C=\frac{\mathrm{d} \Omega^{\prime}}{16 \pi^{2}} W_{\mathrm{A}}^{\gamma} W_{\mathrm{B}}^{\delta} \omega_{\mathrm{A}}^{j} \omega_{\mathrm{B}}^{i} C_{\mathrm{A}} C_{\mathrm{B}} \frac{r_{\mathrm{A}}^{j}-1}{r_{\mathrm{A}}^{j}} \mu_{\mathrm{B}}^{\mathrm{A}}
$$

An explicit calculation of integral (7) assumes the introduction of a mathematically defined geometry. As argued before an infinite wedge with wedge angle $\alpha$ will be chosen.

2.2 APPROXIMATION. - In practice the six dimensional integral (7) can hardly be evaluated numerically. Expression (7) will therefore be reduced to a four dimensional integral by projecting the three dimensional excitation volume $V$ onto the optical axis. The integration over $V$ is then replaced by a one dimensional integration from 0 to $T$ the total mass thickness of the sample along the optical axis (Fig. 1). The final figures shown in this paper and calculated with the four dimensional approximation are the result of approximately 25 days of CPU time on a CDC mainframe CYBER 170-815. The full six dimensional treatment with the same sixpoint Gaussian algorithm would have taken about 3600 times more computing time (or \pm 250 years !).

Another reason why a correct six dimensional treatment is not feasible yet is the absence of a correct analytical expression for the shape and intensity of the primary excitation volume in the case of a wedge shaped specimen arbitrary oriented with respect to the electron beam. However, the qualitative conclusion on the fluorescence behaviour in wedge shaped targets will not be affected by the approximation. It is also worth mentioning that the validity of the approximation improves as the mass thickness $T$ decreases because then the excitation volume $V$ becomes cylindrical around the optical axis. The consequences of a projection onto the optical axis then become negligible.

In the approximation of a one dimensional excitation volume the spatial ionization distribution function $\varphi(r)$ becomes a one dimensional depth distribution function $\varphi(t), t$ being the mass depth. 
$\varphi(t)=1$ is a good approximation as long as the specimen is perfectly transparent for transmission or scanning transmission electron microscopy, but if the influence of a scanty electron scattering has to be accounted for the simple mass depth distribution is used :

$$
\varphi(t)=\exp (-\sigma t)
$$

proposed by several authors (Tixier [1] ; Philibert et al. [2] ). The electron absorption coefficient $\sigma$ has the empirical from (Heinrich [6] ):

$$
\sigma=\frac{4.25 \times 10^{5}}{E_{0}^{1.67}-E_{j}^{1.67}}
$$

Where $E_{0}$ is the operating voltage and $E_{j}$ the minimum excitation potential. This approach might seem inaccurate as it,does not account for the slight initial increase of $\varphi(t)$ due to backscatter effect (Stenton et al. [7] ). However, expression (9) was chosen because of its reasonable computation time, and the fact that comparative results are not affected by the exact shape of the mass depth distribution.

In expression (7) the three dimensional integral over the excitation volume $V$ is replaced by a one dimensional integral over the mass thickness $T$ along the optical axis. To simplify the notation of (7) the element of volume $d V^{\prime}$ can be replaced by $d V$ and $\mathbf{r}-\mathbf{r}$ by $\mathbf{r}$. This new vector $\mathbf{r}$ connects point $P(t)$, on the optical axis at a mass depth $t$, where primary emission of element $\mathrm{B}$ is emitted, with the element of volume $\mathrm{d} V$ where secondary emission of element $\mathrm{A}$ is generated. With these simplified notations expression (7) becomes :

$$
I_{\mathrm{A}}^{\mathrm{F}}=C \int_{0}^{T} \int_{\text {spec }} \exp (-\sigma t) \frac{\exp \left(-\mu_{\mathrm{B}}|\mathbf{r}|\right)}{|\mathbf{r}|^{2}} \exp \left(-\mu_{\mathrm{A}} \cdot q(\mathbf{r}, t)\right) \mathrm{d} V \mathrm{~d} t
$$

where $C$ is still given by (8).

\section{Wedge geometry.}

3.1 COORDINATE SYSTEM. - A reference frame has to be chosen to calculate the boundaries of the three dimensional integral over the specimen volume. The new vector $\mathbf{r}$ was introduced so as to transform the problem to spherical coordinates. The origin and the $Z$-axis of the frame are taken respectively in $\mathrm{P}$ and along the optical axis of the microscope. For an untilted specimen (symmetry plane of the wedge perpendicular to the optical axis) the $X$-axis is chosen parallel to the edge of the wedge and consequently the $Y$-axis is perpendicular to it (Fig. 2). To allow for an arbitrary electron impact direction the specimen is first rotated around the $X$-axis over an angle " $a$ " followed by a rotation around the $Y$-axis over an angle " $b$ ". Performing the rotations in this order leaves the $Y$-axis perpendicular to the edge of the specimen which turns out to be most convenient for the calculations of the integration boundaries.

In spherical coordinates integral (11) reads :

$$
\begin{array}{r}
I_{\mathrm{A}}^{\mathrm{F}}=C \int_{0}^{T} \int_{0}^{2 \pi} \int_{0}^{\pi} \int_{0}^{R(\varphi, \theta, t)} \exp (-\sigma t) \exp \left(-\mu_{\mathrm{B}} r\right) \exp \left(-\mu_{\mathrm{A}} q\right) \\
\cdot \sin \theta \mathrm{d} r \mathrm{~d} \theta \mathrm{d} \varphi \mathrm{d} t
\end{array}
$$

The limit $R(\varphi, \theta, t)$ is the intersection of the position vector $\mathbf{r}$ with the wedge, therefore a mathematical description of the wedge is needed. The upper $(\mathrm{U})$ and lower $(\mathrm{L})$ planes of the wedge will 


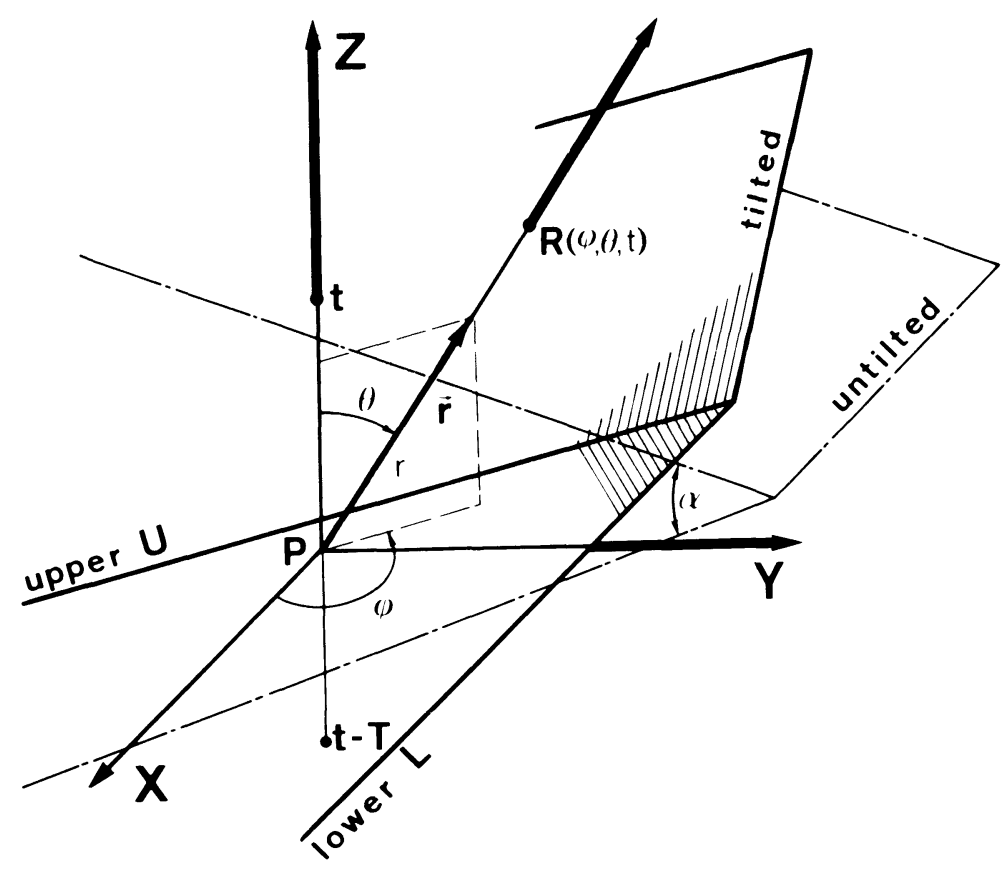

Fig. 2. - Spherical coordinate system. The $Z$-axis is the optical axis of the microscope and for an untilted specimen the $X$-axis is chosen parallel to the specimen edge. A rotation around the $X$-axis followed by one around the $Y$-axis gives the "tilted" orientation. $R(\varphi, \theta, t)$ is the upper boundary of the integral over the variable $r$.

be described by their normal vectors " $n_{+}$" and "n_". For an untilted specimen it is easily seen that the components of these normals are (see Fig. 2):

$$
\begin{aligned}
& \mathbf{n}_{+}=(0, \sin \alpha / 2, \cos \alpha / 2) \\
& \mathbf{n}_{-}=(0, \sin \alpha / 2,-\cos \alpha / 2)
\end{aligned}
$$

The normals of the tilted wedge are found by applying a double rotation transformation (Van Cappellen et al. [8] ) :

$$
\begin{aligned}
& \mathbf{n}_{+}=\cos (\alpha / 2-a)(\sin b, \tan (\alpha / 2-a), \cos b) \\
& \mathbf{n}_{-}=\cos (\alpha / 2-a)(-\sin b, \tan (\alpha / 2+a),-\cos b)
\end{aligned}
$$

The resulting equations for the upper $(\mathrm{U})$ and lower $(\mathrm{L})$ wedge planes are :

$$
\begin{aligned}
& \mathrm{U}: x \sin b+y \tan (\alpha / 2-a)+(2-t) \cos b=0 \\
& \mathrm{~L}: x \sin b-y \tan (\alpha / 2+a)+(2-t+T) \cos b=0
\end{aligned}
$$

3.2 THE UPPER BOUNDARY $R(\varphi, \theta, t)$. $-R(\varphi, \theta, t)$ is the intersection of the position vector $\mathbf{r}$ with the wedge surface. The intersections with $U$ and $\mathrm{L}$, denoted $R_{+}$and $R_{-}$, are found after 
straightforward calculation :

$$
\begin{aligned}
& R_{+}=\left|\frac{r \cdot t \cos b \cdot \cos (\alpha / 2-a)}{\mathbf{r} \cdot \mathbf{n}_{+}}\right| \\
& R_{-}=\left|\frac{r(t-T) \cos b \cdot \cos (\alpha / 2+a)}{\mathbf{r} \cdot \mathbf{n}_{-}}\right|
\end{aligned}
$$

Clearly the boundary $R(\varphi, \theta, t)$ can be either $R_{+}, R_{-}$or infinite depending on the direction of the position vector $\mathbf{r}$. A convenient way to determine the correct $R(\varphi, \theta, t)$ is to check the signs of the scalar products $\mathbf{r} \cdot \mathbf{n}_{+}$and $\mathbf{r} \cdot \mathbf{n}_{-}$. Indeed, if for example the position vector $\mathbf{r}$ "points" towards the upper plane $U, \mathbf{r} \cdot \mathbf{n}_{+}$will be positive and vice versa. When both scalar products are positive the smallest of $R_{+}$and $R_{-}$must be choosen as boundary the other being an unphysical intersection.

These conditions can be translated into actual integration boundaries. If $\mathbf{r} \cdot \mathbf{n}_{+}=0$ for $\theta=\theta_{+}$ and $\mathbf{r} \cdot \mathbf{n}_{-}=0$ for $\theta=\theta_{-}$it becomes clear that :

$$
\begin{aligned}
& \mathbf{r} \cdot \mathbf{n}_{+} \geq 0 \text { when } \theta \in\left[0, \theta_{+}\right] \\
& \mathbf{r} \cdot \mathbf{n}_{-} \geq 0 \text { when } \theta \in\left[\theta_{-}, \pi\right]
\end{aligned}
$$

Since the $Y$-axis is still perpendicular to the specimen edge the two possible cases, $\theta_{-}<\theta_{+}$and $\theta_{-}>\theta_{+}$can easily be distinguished. Indeed for any angle $\varphi$ between 0 and $\pi, \theta_{-}$is smaller than $\theta_{+}$while $\theta_{-}$is larger than $\theta_{+}$when $\varphi$ lies between $\pi$ and $2 \pi$. The situation is represented schematically in figure 3 .
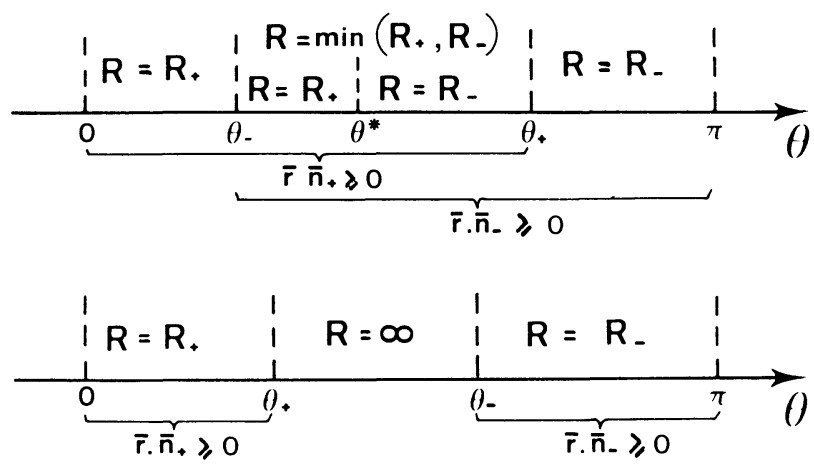

Fig. 3. - The boundary $R$ as a function of $\varphi$ and $\theta$.

For $(\varphi \in[0, \pi])$ the condition " $R=\min \left(R_{+}, R\right)$ " can be simplified since in the interval $\left[\theta_{-}, \theta_{+}\right] R_{+}$increases and $R_{-}$decreases, monotonically, with $\theta$. As a consequence an angle $\theta^{*}$ exists for which $R_{+}=R_{-}$. Below this angle $R_{+}=\min \left(R_{+}, R_{-}\right)$whereas for angles exceeding $\theta^{*}$ $R_{-}=\min \left(R_{+}, R_{-}\right)$.

The analytical expression for $\theta_{+}, \theta_{-}$and $\theta^{*}$ read (Van Cappellen et al. [8] ) :

$$
\begin{aligned}
& \theta_{+}=\operatorname{arcctg}\left[-\frac{\sin b \cos \varphi+\tan (\alpha / 2-a) \sin \varphi}{\cos b}\right] \\
& \theta_{-}=\operatorname{arcctg}\left[-\frac{\sin b \cos \varphi-\tan (\alpha / 2+a) \sin \varphi}{\cos b}\right] \\
& \theta^{*}=\operatorname{arcctg}\left[-\frac{\sin b \cos \varphi-p(t) \sin \varphi}{\cos b}\right]
\end{aligned}
$$


with :

$$
p(t)=(t / T-1) \tan (\alpha / 2-a)+t / T \tan (\alpha / 2+a)
$$

Using the integration boundaries summarized in table I integral (11) becomes :

$$
\begin{array}{r}
I_{\mathrm{A}}^{\mathrm{F}=C \int_{0}^{T} \mathrm{~d} t\left\{\int _ { 0 } ^ { \pi } \mathrm { d } \varphi \left(\int_{0}^{\theta^{*}(\varphi, t)} \mathrm{d} \theta\left(\int_{0}^{R_{+}(\varphi, \theta, t)} \mathrm{d} r I\right)\right.\right.}+\int_{\theta^{*}(\varphi, t)}^{\pi} \mathrm{d} \theta\left(\int_{0}^{R_{-}(\varphi, \theta, t)} \mathrm{d} r I\right) \\
+\int_{\pi}^{2 \pi} \mathrm{d} \varphi\left(\int_{0}^{\theta_{+}(\varphi)} \mathrm{d} \theta\left(\int_{0}^{R_{+}(\theta, \varphi, t)} \mathrm{d} r I\right)+\int_{\theta_{+}(\varphi)}^{\theta_{-}(\varphi)} \mathrm{d} \theta\left(\int_{0}^{\infty} \mathrm{d} r I\right)\right. \\
\left.\left.+\int_{\theta_{-}(\varphi)}^{\pi} \mathrm{d} \theta \cdot\left(\int_{0}^{R(\theta, \varphi, t)} \mathrm{d} r I\right)\right)\right\}
\end{array}
$$

where :

$$
I=\exp (-\sigma t) \exp \left(-\mu_{\mathrm{B}} \cdot r\right) \exp \left(-\mu_{\mathrm{A}} q(\mathbf{r}, t)\right) \sin \theta
$$

3.3 ABSORPTION OF THE SECONDARY EMISSION. - The only unknown left is $q$, the distance over which the secondary emission of element A must propagate through the specimen in the direction of the detector. To calculate $q$, the detector direction must be specified to define the line $\mathrm{P}_{1} \mathrm{P}_{2}$; $P_{1}$ is the point where the secondary emission is generated and $P_{2}$ the point where this radiation leaves the specimen in the direction of the detector (see Fig. 4). $q$ can be calculated from the coordinates of the points $\mathrm{P}_{1}$ and $\mathrm{P}_{2}$ with coordinates (Van Cappellen et al. [8] ) :

$$
\begin{aligned}
& \mathrm{P}_{1}:\left\{\begin{array}{l}
x_{1}=r \sin \theta \cos \varphi \\
y_{1}=r \sin \theta \sin \varphi \\
z_{1}=r \cos \theta
\end{array}\right. \\
& \mathrm{P}_{2}:\left\{\begin{array}{c}
x_{2}=q[r(k(\cos b+\tan \Theta \sin \Phi \tan (\alpha / 2-a))-\ell \tan \Theta \cos \Phi \tan (\alpha / 2-a)) \\
\quad+t \cos b \tan \Theta \cos \Phi] \\
y_{2}=q[-r(k \cdot \tan \Theta \sin \Phi \sin b-l(\cos b+\sin b \tan \Theta \cos \Phi)) \\
z_{2}=q[-r(k \sin b+l \tan (\alpha / 2-a))+t \cos b]
\end{array}\right.
\end{aligned}
$$

where :

$$
\begin{aligned}
k & =\sin \theta \cos \varphi-\cos \theta \tan \Theta \cos \Phi \\
l & =\sin \theta \sin \varphi-\cos \theta \tan \Theta \sin \Phi \\
q & =1 /[\cos b+\tan \Theta \sin \Phi \tan (\alpha / 2-a)+\sin b \tan \Theta \cos \Phi]
\end{aligned}
$$

and :

$$
q=\left[\left(x_{1}-x_{2}\right)^{2}+\left(y_{1}-y_{2}\right)^{2}+\left(z_{1}-z_{2}\right)^{2}\right]^{1 / 2}
$$

\section{Computer simulations.}

4.1 INTEGRATION. - Obviously the integral (25)-(26) cannot be solved analytically. All integrations are performed by means of a sixpoint Gaussian method. The graphs shown below represent the secondary emission of element $\mathrm{A}$ as a function of the specimen thickness along the optical axis. A number of different situations are examined. The curves are obtained by calculating the secondary emission at different thicknesses and a least square fit is used to connect the calculated points. Two distinct thickness sequences are used. 


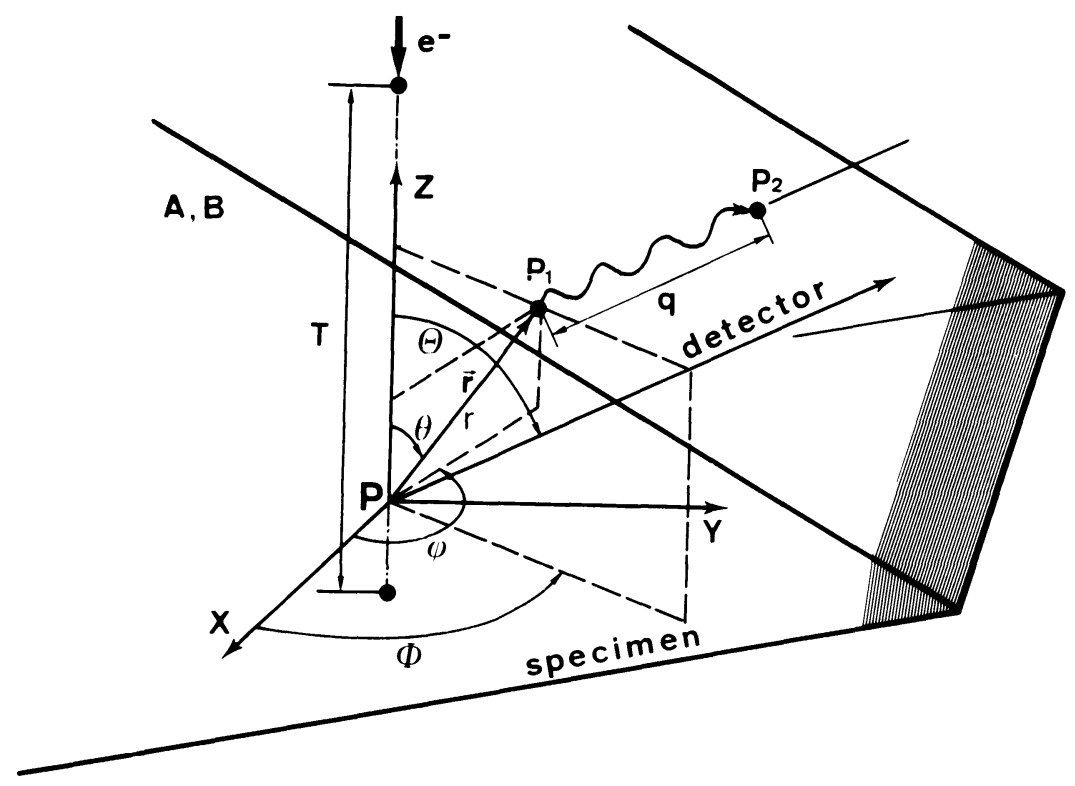

Fig. 4. - Attenuation of the secondary emission due to absorption in the specimen can be accounted for by calculating " $q$ " the distance to be covered by the secondary $X$-ray photons in the specimen. $q$ is a fonction of the position vector $r(r, \varphi, \theta)$, the detector orientation $(\Theta, \Phi)$ the mass depth $t$ and the specimen geometry.

For the figures from 0 to $100 \mathrm{~nm}$ and from 0 to $500 \mathrm{~nm}$ the sequence is : $0.5,1,3,6,10,30,60$, $100,150,200,300$ and $500 \mathrm{~nm}$. For the graphs up to 5000 or $10000 \mathrm{~nm}$ the thickness sequence is : $10,100,1000,2000,3000, \ldots, 9000$ and $10000 \mathrm{~nm}$. The figures do not account for the constant $C$ in equation (7).

The calculations are carried out for an alloy : $\mathrm{Cu} 50$ at $\%-\mathrm{Cr}$. The density of the material is approximately $8 \mathrm{~g} / \mathrm{cm}^{3}$ while the mass absorption coefficients of the copper and chromium peaks in the considered alloy are respectively equal to: $\mu_{\mathrm{A}}=\mu_{\mathrm{Cr}}=120 \mathrm{~cm}^{2} / \mathrm{g} ; \mu_{\mathrm{B}}=\mu_{\mathrm{Cu}}=152 \mathrm{~cm}^{2} / \mathrm{g}$ The first graph shows the influence of the absorption of the secondary emission of element $A$ and of the deceleration of the electrons in the specimen. The curves of figure 5 are calculated for a wedge shaped target with a wedge angle of $30^{\circ}$. Both absorption phenomena are neglected in the dashed curve $\left(\sigma=0 ; \mu_{\mathrm{A}}=0\right)$. The dash-dot curve accounts for the absorption of the fluorescence emission $\left(\sigma=0 ; \mu_{\mathrm{A}}=120 \mathrm{~cm}^{2} / \mathrm{g}\right)$ and the full curve is the result of a complete treatment $\left(\sigma=200 \mathrm{~cm}^{2} / \mathrm{g} ; \mu_{\mathrm{A}}=120 \mathrm{~cm}^{2} / \mathrm{g}\right)$. The numerical value of $\sigma$ corresponds with an operating voltage of $100 \mathrm{kV}$. Since the influences of both absorption phenomena are not negligible, although they only become very important above the transparancy limit $( \pm 500 \mathrm{~nm})$, they are taken into account in all further calculations.

In the second set of graphs (Figs. 6a and $6 \mathrm{~b}$ ) the influence of the operating voltage is investigated. The relationship between $\sigma$ and the operating voltage is given by equation (10). Again the curves are calculated for a wedge of $30^{\circ}$. From figure $6 \mathrm{a}$ the strong accelerating voltage dependence is clearly seen. At $20 \mathrm{kV}$ the specimen can already be considered as infinitely thick at $2000 \mathrm{~nm}$. Figure $6 \mathrm{~b}$ is a detail of figure $6 \mathrm{a}$ and covers the thickness range of interest in transmission electron microscopy. Above $60 \mathrm{kV}$ the accelerating voltage has nearly no influence on the 


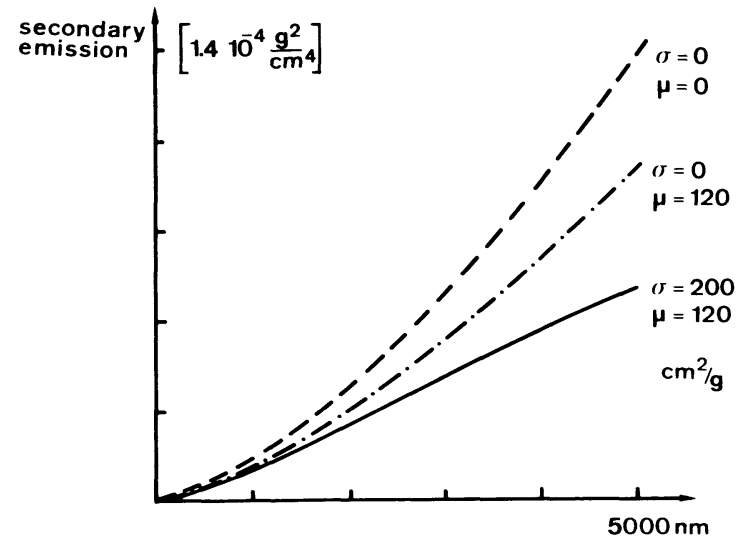

Fig. 5. - Calculations of secondary emission for a $\mathrm{Cu} 50$ at\% $-\mathrm{Cr}$ wedge with a wedge angle of $30^{\circ}$. The dashed curve neglects absorption of the secondary $\mathrm{Cr}-\mathrm{K} \alpha$ emission and the deceleration of the electrons in the target. The dash-dot curve takes account of the absorption of the secondary $-\mathrm{K} \alpha$ radiation and the full curve of both absorption phenomena. $\alpha=30^{\circ}, a=-30^{\circ} ; b=0^{\circ} ; \Phi=\Theta=90^{\circ}$.

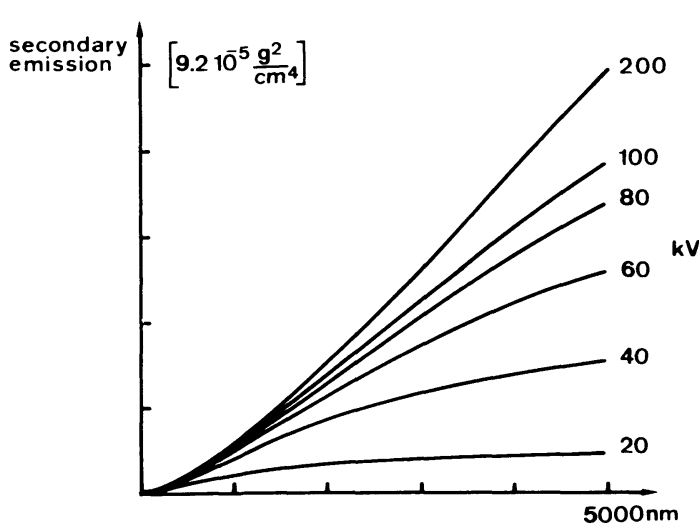

(a)

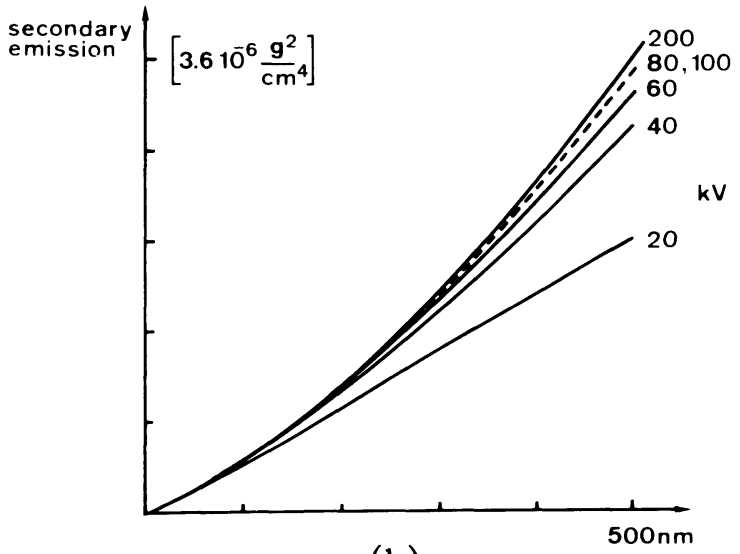

(b)

Fig. 6. - Calculations of the fluorescence intensity as a function of thickness for various accelerating voltages. $\alpha=30^{\circ}, a=-30^{\circ} ; b=0^{\circ} ; \Phi=\Theta=90^{\circ}$ : a) from 0 to $5000 \mathrm{~nm}$; b) from 0 to $500 \mathrm{~nm}$.

fluorescence intensity which also proves that the model for the ionization distribution function $\varphi(t)$ is not very critical.

The influence of the wedge angle $\alpha$ is investigated in figures 7a and 7b. 7a covers the transparancy thickness range and the curves are calculated for wedge angles of $0^{\circ}$ (plane parallel foil) up to $80^{\circ}$ and an accelerating voltage of $100 \mathrm{kV}$. The dramatic increase in fluorescence emission with increasing wedge angle is emphasized in figure $7 \mathrm{~b}$ which covers the most important thickness range. For instance it appears that a thickness of $50 \mathrm{~nm}$ the secondary emission is twice as important for a wedge of $10^{\circ}$ than for a plane parallel foil whereas for a wedge of $40^{\circ}$ this in- 
tensity is already six times as large. In other words, modest changes in the shape of the specimen have a dramatic effect on the secondary emission intensity.

From the least square fits it may be derived that the linear term of the curve is directly proportional to $\alpha$. As a consequence the fluorescence curve for a plane parallel foil $\left(\alpha=0^{\circ}\right)$ starts as a pure parabola. This $T^{2}$ dependence is in perfect agreement with the formula of Tixier [1] and Philibert et al. [2] . Higher order terms will bend the curve towards the abscis.

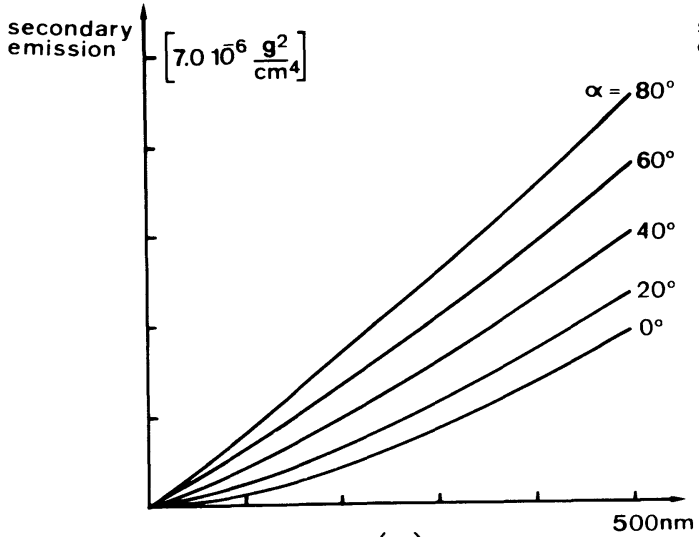

(a)

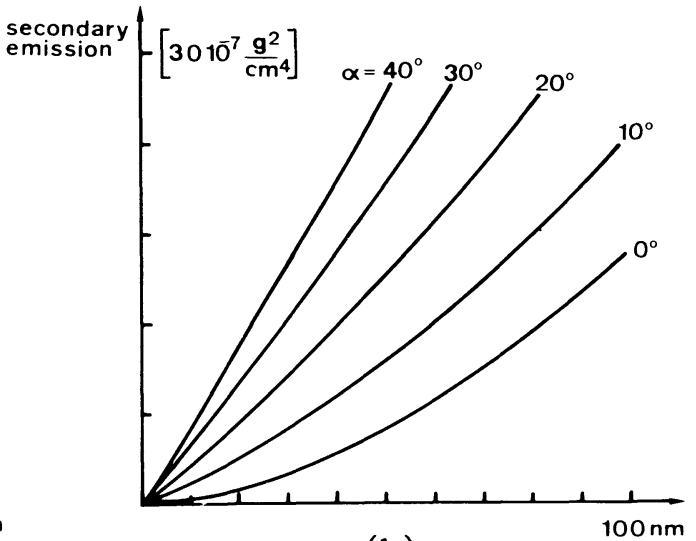

(b)

Fig. 7. - The fluorescence intensity as a function of thickness for various wedge angles $\alpha$. $\sigma=$ $200 \mathrm{~cm}^{2} / \mathrm{g}(100 \mathrm{kV}) ; a=-30^{\circ} ; b=0^{\circ} ; \Phi=\Theta=90^{\circ}$ : a) from 0 to $500 \mathrm{~nm}$; b) from 0 to $100 \mathrm{~nm}$.

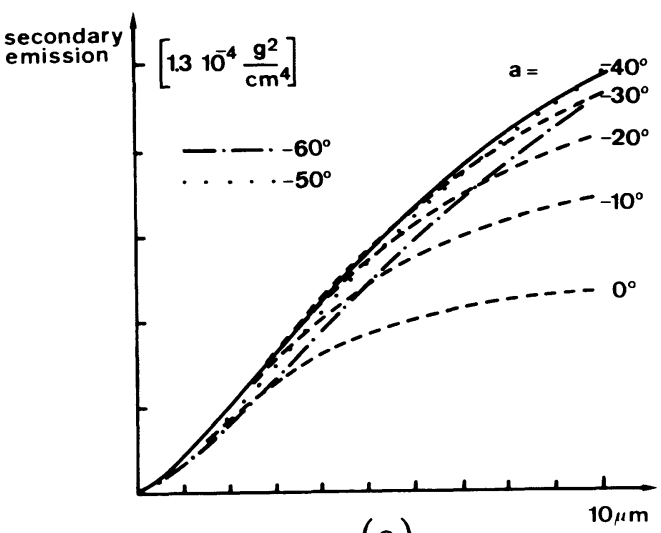

(a)

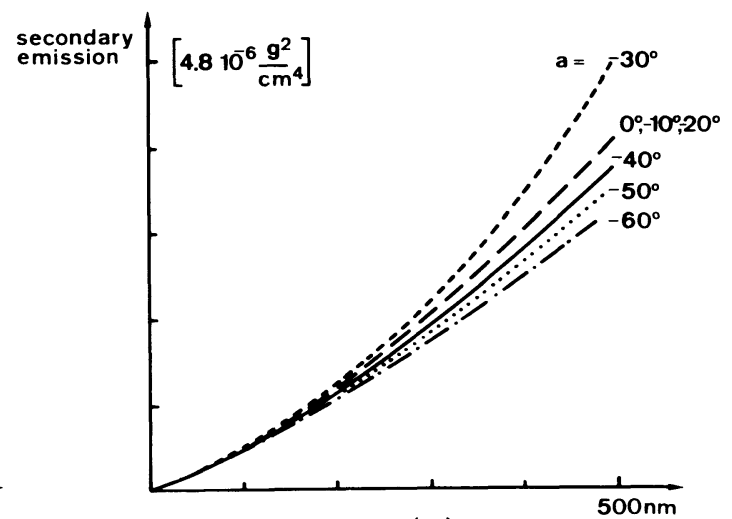

(b)

Fig. 8. - The fluorescence intensity as a function of thickness for different rotations around the $X$-axis (Fig. 2). Negative $a$ angles correspond to rotations towards the detector of the upper plane. $\alpha=30^{\circ} ; \sigma=$ $200 \mathrm{~cm}^{2} / \mathrm{g}(100 \mathrm{kV}) ; b=0^{\circ} ; \Phi=\Theta=90^{\circ}$. a) from 0 to $\left.10 \mu \mathrm{m} ; \mathrm{b}\right)$ from 0 to $500 \mathrm{~nm}$. 


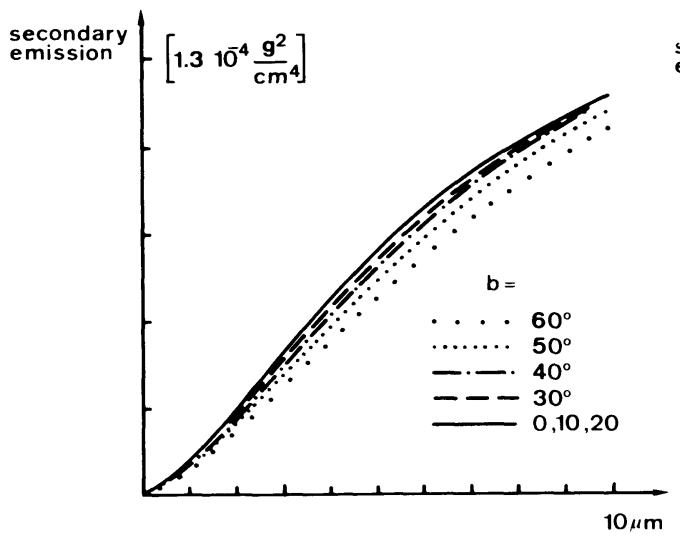

(a)

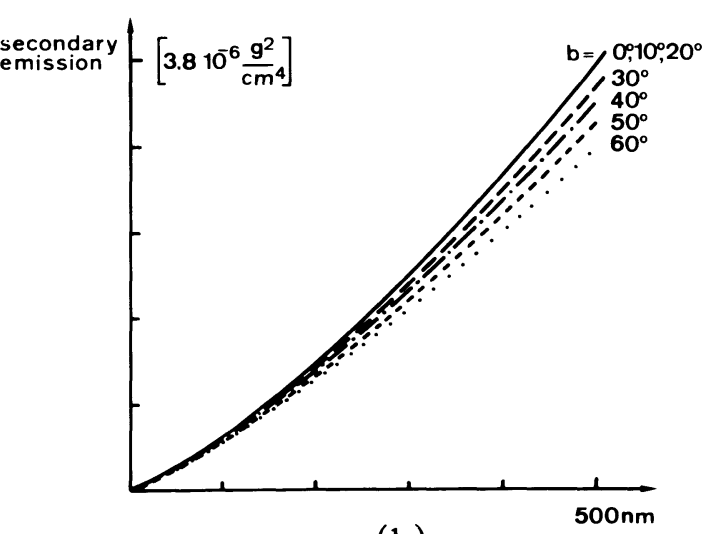

(b)

Fig. 9. - The fluorescence intensity as a function of thickness for different rotations around the $Y$-axis (Fig. 2) For $\Phi=90^{\circ}$ no distinction can be made between positive and negative b angles. $\alpha=30^{\circ} ; \sigma=$ $\left.200 \mathrm{~cm}^{2} / \mathrm{g}(100 \mathrm{kV}) ; a=-30^{\circ} ; \Phi=\Theta=90^{\circ}: \mathrm{a}\right)$ from 0 to $10 \mu \mathrm{m}$; b) from 0 to $500 \mathrm{~nm}$.

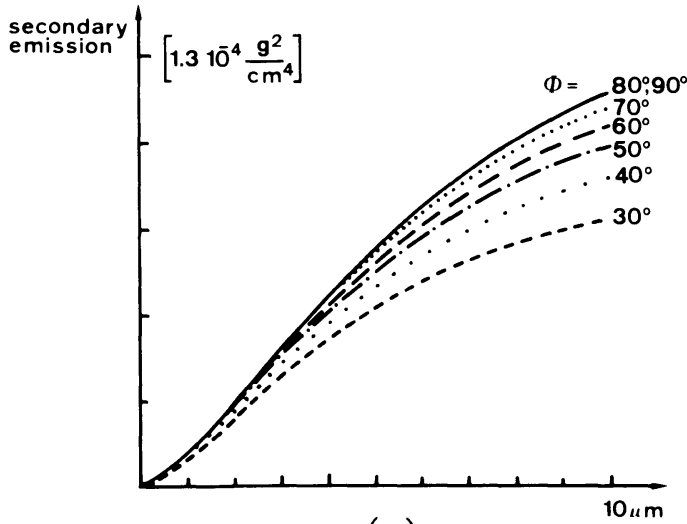

(a)

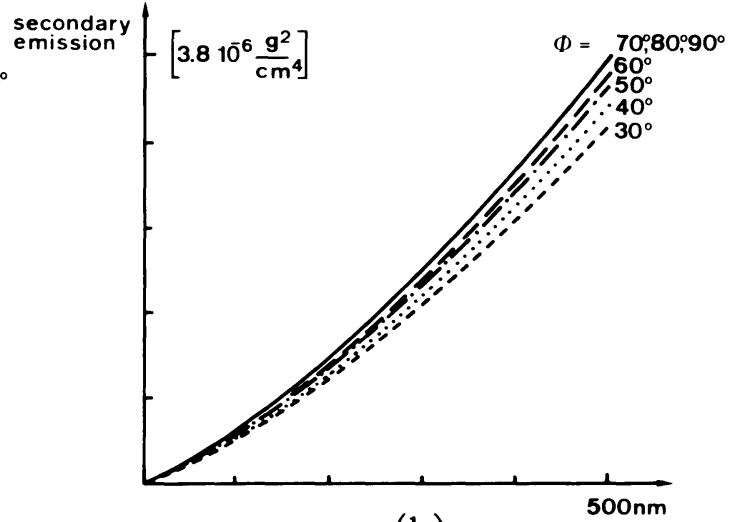

(b)

Fig. 10. - The fluorescence intensity as a function of thickness for different detection angles $\Phi$ (Fig. 3). $\alpha=30^{\circ} ; \sigma=200 \mathrm{~cm}^{2} / \mathrm{g}(100 \mathrm{kV}) ; a=-30^{\circ} ; b=0^{\circ} ; \Theta=90^{\circ}:$ a) from 0 to $\left.10 \mu \mathrm{m} ; \mathrm{b}\right)$ from 0 to $500 \mathrm{~nm}$.

The influences of specimen orientation (the angles $a$ and $b$ ) and of the detection direction (the angles $\Theta$ and $\Phi$ ) are investigated in the figure 8a up to 11b. Again all the curves are calculated for a wedge angle of $30^{\circ}$ and an accelerating voltage of $100 \mathrm{kV}$. The conclusion is that for thickness within the transparancy limit neither the orientation of the specimen nor the detection direction substantially affect the secondary emission intensity. 


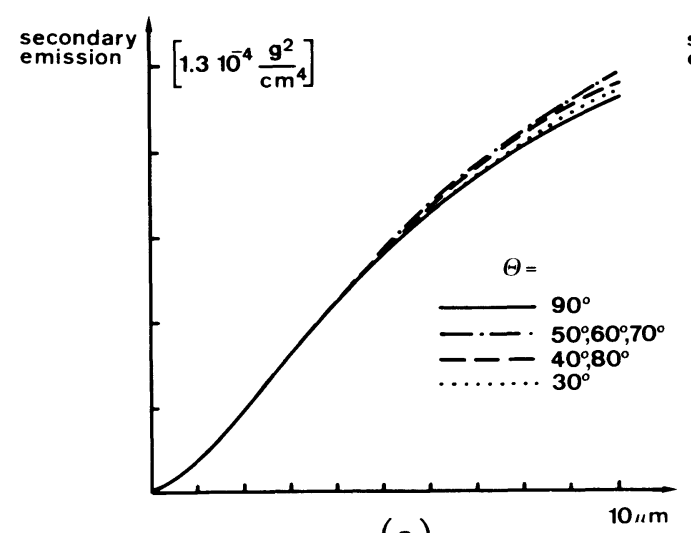

(a)

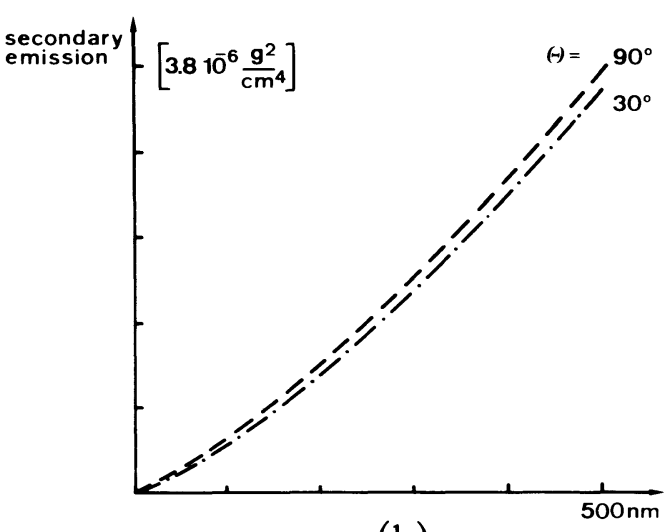

(b)

Fig. 11. - The fluorescence intensity as a function of thickness for different detection angles $\Theta$ (Fig. 3). $\left.\alpha=30^{\circ} ; \sigma=200 \mathrm{~cm}^{2} / \mathrm{g}(100 \mathrm{kV}) ; a=-30^{\circ} ; b=0^{\circ} ; \Phi=90^{\circ}: \mathrm{a}\right)$ from 0 to $\left.10 \mu \mathrm{m} ; \mathrm{b}\right)$ from 0 to $500 \mathrm{~nm}$.

\section{Conclusions.}

Especially the wedge angle $\alpha$, a newly introduced parameter turns out to be very important since it considerably influences the fluorescence yield. Computer simulations clearly demonstrate the influence of the real specimen shape on the secondary X-ray emission. Therefore it is our conviction that no fluorescence correction can be entirely satisfactory unless the exact shape of the specimen is introduced in the correction procedure.

Since X-rays travel considerable distances in the specimen when compared with the typical dimensions of the primary excitation volume, the secondary excitation volume will be some orders of magnitude larger than the primary one. Consequently the shape should not only be known in the neighbourhood of the electron irradiated area but in a much larger area, typically some tenths of a millimeter in diameter or even more. Even if a fluorescence correction procedure based on a "fast" on-line computer simulation for an arbirary target geometry could be developed in the future it remains extremely doubtful whether it will be feasible routinely to determine the exact shape of the specimen.

The computer simulations also confirm the three dimensional aspect of fluorescence. Indeed, neither the orientation of the specimen in the electron beam nor the detection direction seem to have a noticeable effect on fluorescence emission which is mainly governed by the three dimensional geometry of the specimen. This conclusion is in contrast with the correction for the absorption of primary X-radiation, a two dimensional phenomenon confined to the optical axis-detector axis plane and very sensitive to the orientation of the specimen and the detection direction.

\section{References}

[1] TIXIER R., Ph. D., Thesis Université Paris-Sud (1973) Série A, nº 196.

[2] PhILIBERT J. and TIXIER R., in Physical Aspects of Electron Microscopy and Microbeam Analysis, Eds. B. Siegel and D. Beaman (J. Wiley and Sons) 1975, p. 333. 
[3] STATHAM P. J., J. Microsc. 123 (1981) 1.

[4] Nockolds C., NASIR M. J., Cliff G. and Lorimer G. W., Inst. Phys. Conf. Series 52 (1980) 417.

[5] Van CAPPEllen E., Ph.D. Thesis, University of Antwerp, UIA, Belgium (1986).

[6] HEINRICH K.F.J., Adv. X-ray Anal. 11 (1968) 40.

[7] STENTON N., Notis M. R., GoldSTEIN J. I. and Williams D. B., in Quantitative Microanalysis with high spatial resolution (The Metals Society, London) 1981, p. 35.

[8] Van Cappellen E., Deblieck R., Van LanduYt J. and AdaMs F., J. Trace Microprobe techniques 2 (1984) 139.

[9] VAN CAPPELlen E., Microsc. Microanal Microstruct. 1 (1990) 1.

Cet article a été imprimé avec le Macro Package "Editions de Physique Avril 1990". 